\title{
Estimation of Small Unmanned Aerial Vehicle Lateral Dynamic Model with System Identification Approaches
}

\author{
E. BELGE, H. K. KABA, A. PARLAK, A. ALTAN, R. HACIOĞLU
}

\begin{abstract}
Modeling of unmanned aerial vehicle (UAV) with system identification is very important in terms of its model-based effective control. The modeling of $\mathrm{UAV}$ is required for aircraft crashes, analyzing autonomous aircrafts, preventing external disturbances, pre-flight analysis. However, since UAV has nonlinear inherent dynamics including inherent chaoticity and fractality, it becomes difficult to obtain a mathematical model under external disturbance. In this study, some of the inherent nonlinear dynamics of UAV are linearized and the model of UAV is obtained by system identification approaches under external disturbance. The linearized lateral dynamics of a fixed wing UAV is used in this study. Further, the flight motion equations applied to fixed wing UAV have been utilized for obtaining the coefficients of lateral model for straight and level flight. The roll angles are calculated using transfer functions for aileron, rudder and deflections inputs. The autoregressive exogenous (ARX), autoregressive moving average with exogenous (ARMAX) and output error (OE) parametric system identification approaches are performed to estimate UAV lateral dynamic system response as using empirical input-output data sets. The accuracy of parametric model estimation and model degrees are compared for different external disturbance effects.
\end{abstract}

Index Terms - System identification, UAV, lateral dynamic model, parameter estimation, external disturbance.

EGEMEN BELGE, is with Department of Electrical Electronics Engineering, Zonguldak Bülent Ecevit University, Zonguldak, Turkey, (e-mail: egemenbelge@beun.edu.tr).

(iD) https://orcid.org/0000-0001-5852-1085

HIZIR KAAN KABA, is with Department of Electrical Electronics Engineering, Zonguldak Bülent Ecevit University, Zonguldak, Turkey, (e-mail: hkaankaba@gmail.com).

(iD) https://orcid.org/0000-0001-5203-8092

AHMET PARLAK, is with Department of Electrical Electronics Engineering, Zonguldak Bülent Ecevit University, Zonguldak, Turkey, (e-mail: mimikli6167@gmail.com).

(iD) https://orcid.org/0000-0003-0751-2263

AYTAC ALTAN, is with Department of Electrical Electronics Engineering, Zonguldak Bülent Ecevit University, Zonguldak, Turkey, (e-mail: aytacaltan@ beun.edu.tr ).

https://orcid.org/0000-0001-7923-4528

RIFAT HACIOĞLU, is with Department of Electrical Electronics Engineering, Zonguldak Bülent Ecevit University, Zonguldak, Turkey, (e-mail: hacirif@beun.edu.tr).

https://orcid.org/0000-0002-2480-0729

Manuscript received Dec 03, 2019; accepted Feb 17, 2020.

DOI: $10.17694 /$ bajece.654499

\section{INTRODUCTION}

Untors NMANNED AERIAL VEHICLE (UAV) modeling has become an important research subject in recent years. Modeling enables analysis of UAV's flight system under different conditions and determination of system reliability. System identification techniques are frequently used in modeling of dynamic systems such as UAV [1]. In this study, the model parameters of UAV is obtained with system identification approaches including Autoregressive Exogenous (ARX), Autoregressive Moving Average with Exogenous Variable (ARMAX) and Output Error (OE) under different external disturbances.

In the literature, there are studies conducted with various system identification methods in the modeling of UAV. In [2], an adaptive system identification technique has been used in real-time dynamic modeling of UAV. For a small UAV, an appropriate approach is proposed, which deals with the online prediction in the frequency domain. In [3], transfer function model with pitch and roll response of UAV are investigated using system identification techniques. The practical system identification procedure of the fixed-wing UAV is specified in [4]. It is emphasized that the system identification procedure used minimize the complexity of UAV movements. In [5], both online and offline models of nonlinear and complex UAV have been obtained using system identification procedure based on Artificial Neural Network (ANN). In [6], the flight control optimization has been handled together with the model of UAV. It is mentioned that hardware installation, flight test, flight data collection and processing, system identification, model verification, controller optimization and verification process steps have been performed. In [7], Hammerstein model is obtained for route tracking under zero averaged white Gaussian noise external disturbing effect based on experimentally obtained input and output data of three-axis camera system on the autonomously acting six-rotor UAV. In [8], the performance of the obtained model has been compared with Nonlinear Autoregressive and Moving Average (NARMA) model performance. The transfer function of the three-axis gimbal system has been obtained by linearly structured $\mathrm{OE}$ model using experimentally obtained data under different external disturbance effect. Model degree has been determined and data set based verification has been applied. Also, the performance has been compared by examining the effect of 
external disturbance in the transfer function obtained. In [9], the model of Load Transporting System (LTS) originally designed on UAV has been obtained by linear ARX model structure and the Model Predictive Controller has been performed. In [10], the Multi Input Multi Output (MIMO) model of helicopter is obtained using ARX system identification model. The flight dynamics of the helicopter have been analyzed with various transfer functions. The system identification of a quadrotorbased aerial manipulator is presented in [11]. ARX and ARMAX models have been obtained from linear accelerations and yaw angular accelerations. The different control-oriented models of a quadrotor UAV have been obtained by applying different identification methods in [12]. Parametric techniques, neural networks, neuro-fuzzy inference systems and hybridization of some of them have been applied. The system identification techniques are utilized in the literature for UAV dynamics. The difference of this study from studies in literature is that the parametric system identification techniques are used for modelling roll angle with aileron and rudder input in fixed wing UAV under external disturbances. The proposed system identification model is more robust to external disturbance variances different from literature studies.

In this study, system identification method is used to define the roll angle for aileron and rudder inputs. The modelling of roll angle is substantial property to define lateral dynamics of small fixed-wing UAV. The OE model proposed to allow the UAV to track the specified route with minimum error is compared with other system identification approaches. This paper is organized as follows: small fixed-wing UAV is modelled, and ARX, ARMAX, OE system identification procedure is explained in Section II. The estimation results of UAV's lateral dynamic model are presented in Section III. Conclusions are finally given in Section IV.

\section{MATERIALS AND METHOD}

\section{A. Fixed-Wing UAV Modelling}

The lateral dynamic model of fixed wing UAV in Fig. 1 is discussed as in [2] where five states $x=(v, p, r, \varphi, c)$, two inputs $u=\left(\delta_{a}, \delta_{r}\right)$, and five outputs $(\beta, p, r, \varphi, c)$ are given.

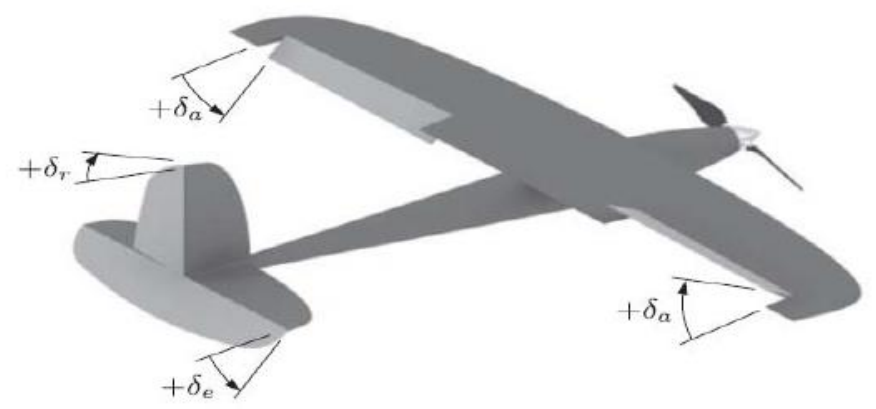

Fig.1. Control surface of fixed wing UAV.

The velocity, roll angular rate, yaw angular rate, roll, yaw, sideslip angle, aileron deflection, rudder deflection parameters are represented by $v, p, r, \varphi, c, \beta, \delta_{a}$, and $\delta_{r}$, respectively. The linearized lateral state space equations can be written as in [13]

$$
\begin{aligned}
& \dot{x}=A x+B u \\
& y=C x
\end{aligned}
$$

where state matrices

$$
\begin{aligned}
& A=\left[\begin{array}{ccccc}
-0.8726 & 0.8789 & -16.82 & 9.791 & 0 \\
-2.823 & -16.09 & 3.367 & 0 & 0 \\
0.702 & 0.514 & -2.775 & 0 & 0 \\
0 & 1 & 0.05406 & -4.088 * 10^{-24} & 0 \\
0 & 0 & 1.001 & -7.573 * 10^{-23} & 0
\end{array}\right] \\
& B=\left[\begin{array}{ccc}
-0.8726 & 5.302 \\
-156.5 & -5.008 \\
11.5 & -82.04 \\
0 & 0 \\
0 & 0
\end{array}\right]
\end{aligned}
$$$$
C=\left[\begin{array}{ccccc}
0.05882 & 0 & 0 & 0 & 0 \\
0 & 1 & 0 & 0 & 0 \\
0 & 0 & 1 & 0 & 0 \\
0 & 0 & 0 & 1 & 0 \\
0 & 0 & 0 & 0 & 1
\end{array}\right]
$$

Transfer function for linearized roll $(\phi)$ output due to aileron $\left(\delta_{a}\right)$ is given as

$$
\frac{\emptyset(s)}{\delta \mathrm{a}(\mathrm{s})}=\frac{-155.8 s^{3}-525.8 s-4283}{s^{4}+19.74 s^{3}+90.49 s^{2}+502.2 s+6.89}
$$

Also the transfer function for linearized roll $(\phi)$ output due to rudder $\left(\delta_{r}\right)$ is given as

$$
\frac{\emptyset(s)}{\delta \mathrm{r}(\mathrm{s})}=\frac{-9.443 s^{3}-384.4 s-4370}{s^{4}+19.74 s^{3}+90.49 s^{2}+502.2 s+6.89}
$$

The roll output data of dynamic lateral system are obtained with normal distribution aileron and rudder inputs. The obtained data is used in parametric estimation process. Both transfer function can be easily discretised by using appropriate sampling time.

\section{B. System Identification Approaches}

The system identification is a phenomenon to construct the mathematical modelling of dynamic systems using measured input output data [14]. There are different type of linear model parametric system identification techniques such as ARX, ARMAX and OE. In this study, the roll output, aileron and rudder input data used the model identification procedure and least-squares based method used to estimate the parameters. The block diagram of parameter estimation process of used system in this study is shown in Fig. 2.

General model structure of discrete-time input-output linear dynamic system with disturbance can be represented as

$$
A(q) y(n)=\frac{B(q)}{F(q)} u(n)+C(q) e(n)
$$

where $u(n), y(n)$ and $e(n)$ are the input, output and disturbance respectively, Also $A(q), B(q), C(q)$ and $F(q)$ are polynomial with the delay operator $q^{-1}$. 


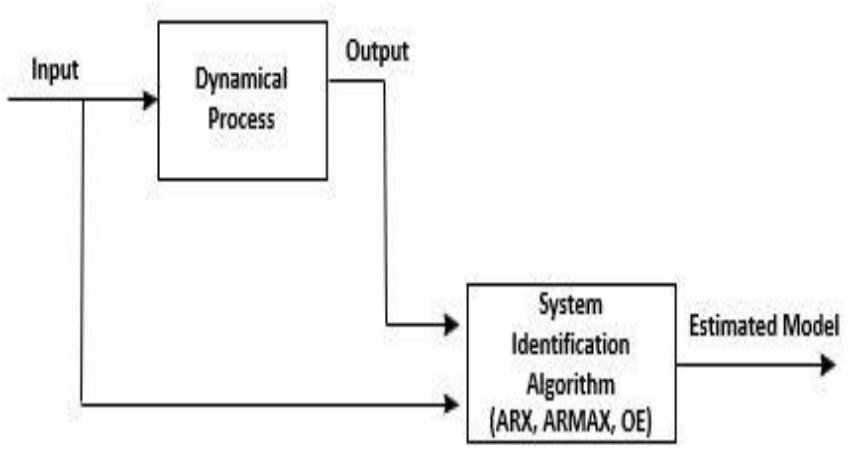

Fig.2. System identification block diagram.

The structure of ARX, ARMAX and OE models are presented in Figs. 3, 4 and 5, respectively.

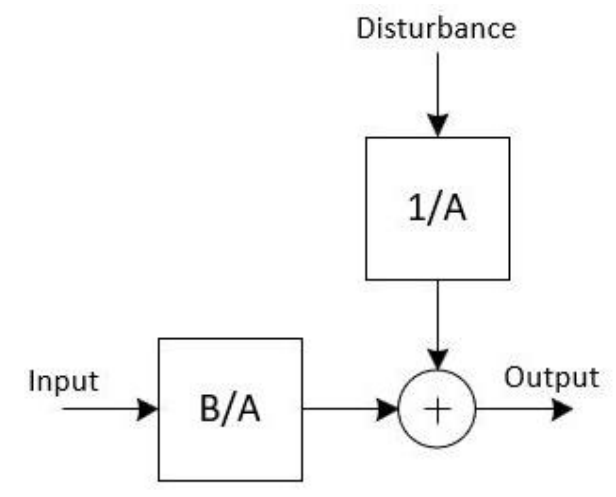

Fig.3. ARX model.

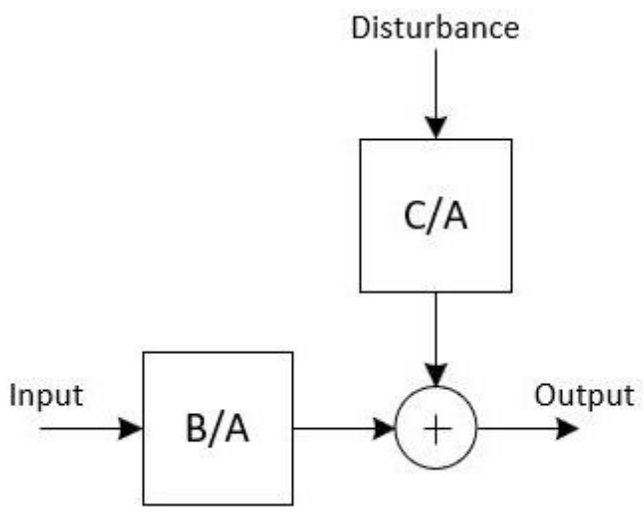

Fig.4. ARMAX model.

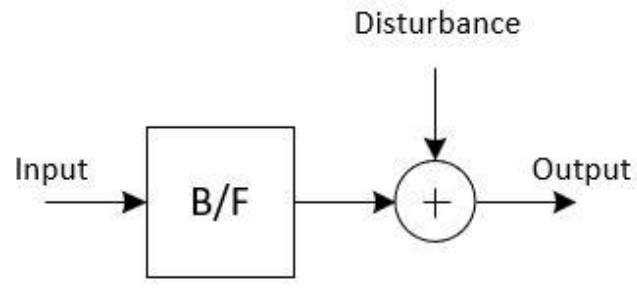

Fig.5. OE model.

$$
\begin{aligned}
& A(q)=1+a_{1} q^{-1}+a_{2}+\ldots+a_{n a} q^{-n a} \\
& B(q)=b_{1} q^{-1}+\ldots+b_{n b} q^{-n b}
\end{aligned}
$$

where model degree is $n a, q^{-1}$ is time delay operator. ARMAX model parameters $a_{k}, b_{k}, c_{k}$ are presented as in Eqs. (6) and (7).

$$
C(q)=1+c_{1} q^{-1}+\ldots+c_{n c} q^{-n c}
$$

ARMAX model includes $C(q)$ parameter unlike ARX model. Model degree $n f$ OE model parameters are indicated in Eqs. (7) and (9).

$$
F(q)=1+f_{1} q^{-1}+\ldots+f_{n f} q^{-n f}
$$

\section{RESULTS AND DISCUSSION}

The input-output data of fixed wing UAV with 0.2 disturbance variance is shown in Fig. 6 for $100 \mathrm{sec}$ normally distribution aileron input where the sampling time $0.1 \mathrm{sec}$. Model order has been changed four to six to get the performance of linear ARX, ARMAX and OE identification approaches. The input output data is divided into test data with the first 700 samples of 1000 samples and validation data with the last 300 samples.
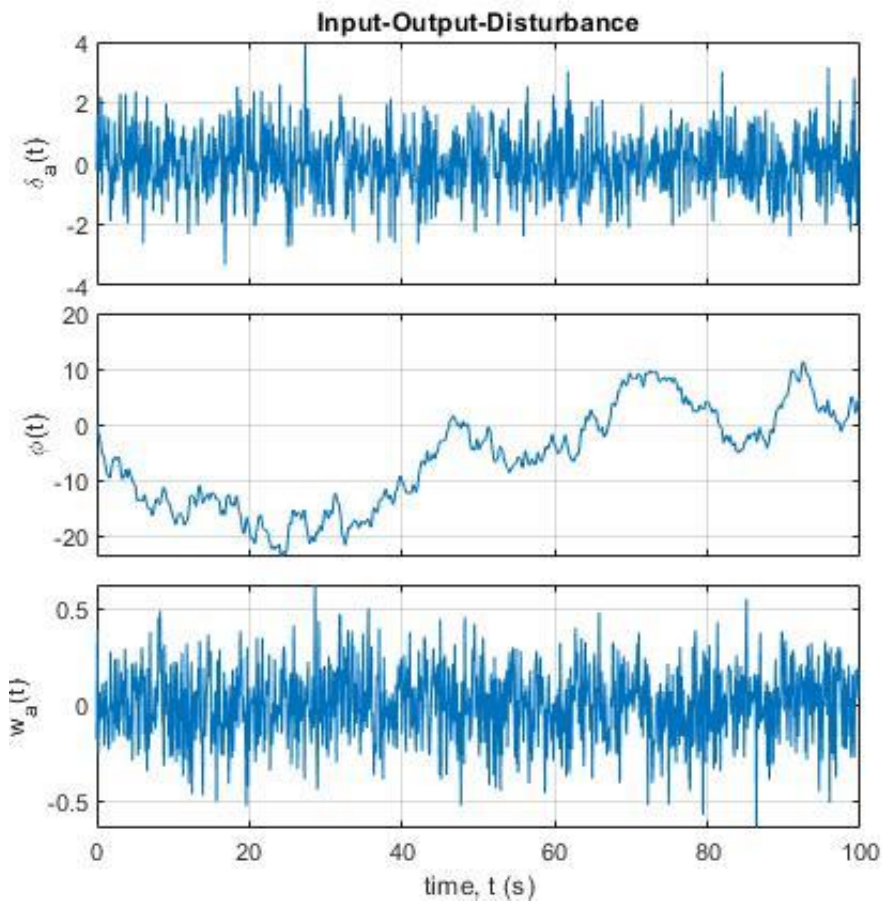

Fig.6. The input-output of fixed-wing UAV with disturbance for aileron input.

The estimated model outputs and squared errors for six model degrees and aileron input are shown in Fig. 7. These graphs express that the OE model accuracy is higher than the other models. The OE and ARMAX model are closer to the system output.

ARX model parameters $a_{k}, b_{k}$ are given as 

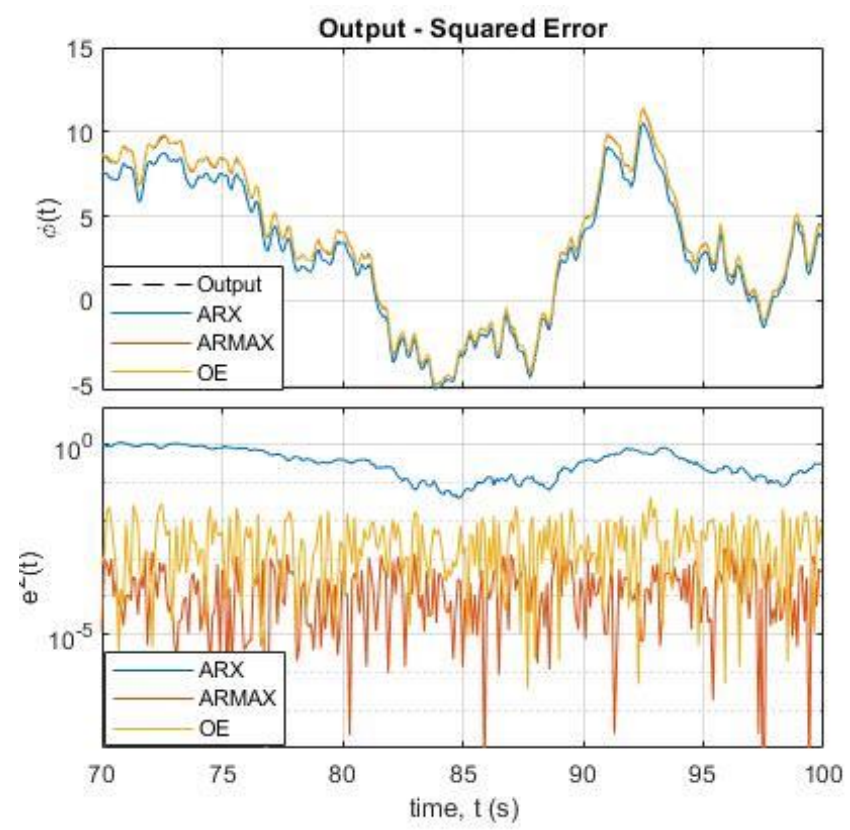

Fig.7. Model outputs and squared errors for aileron input.

The degree of models and estimation ratio system outputaileron input with/without (w/wo) disturbance variance are presented in Table 1.

TABLE 1

DEGREE OF MODELS AND ESTIMATION RATIO w/wo DISTURBANCE FOR AILERON INPUT

\begin{tabular}{c|c|c|c|}
\multirow{2}{*}{ Model Type } & $\begin{array}{c}\text { Model Degree } \\
\text { ARX }(\boldsymbol{n} \boldsymbol{a}, \boldsymbol{n b}) \\
\text { ARMAX }(\boldsymbol{n a}, \boldsymbol{n b}, \boldsymbol{n c})\end{array}$ & \multicolumn{2}{|c|}{$\begin{array}{c}\text { Estimation Ratio } \\
\text { w/wo Disturbance } \\
\text { OE }(\boldsymbol{n} \boldsymbol{b}, \boldsymbol{n} \boldsymbol{f})\end{array}$} \\
\hline ARX & $(4,4)$ & 84.78 & 91.33 \\
\hline ARMAX & $(4,4,4)$ & 86.06 & 97.10 \\
\hline OE & $(4,4)$ & 86.22 & 97.15 \\
\hline ARX & $(5,5)$ & 85.23 & 94.11 \\
\hline ARMAX & $(5,5,5)$ & 86.52 & 99.12 \\
\hline OE & $(5,5)$ & 86.57 & 99.47 \\
\hline ARX & $(6,6)$ & 86.34 & 96.33 \\
\hline ARMAX & $(6,6,6)$ & 86.65 & 99.58 \\
\hline OE & $(6,6)$ & 87.81 & 99.66 \\
\hline
\end{tabular}

The maximum parameter estimation accuracy of system without disturbance for aileron input obtained for $(n f=6, n b=6)$ OE model. The accuracy value of OE model is $99.66 \%$. Further, the maximum accuracy for system with disturbance is obtained for ( $n a=6, n b=6, n c=6)$ ARMAX and $(n f=6, n b=6)$ OE model. The accuracy values of ARMAX and OE model are $86.65 \%$ and $87.81 \%$, respectively.

The rudder input, system output and disturbance graphs are shown in Fig. 8. The disturbance variance is defined as 0.2. The estimated model outputs and squared errors for 6 model degrees and rudder input are shown in Fig. 9. These model error and model output graphs state that the OE and ARMAX model accuracy is higher than ARX model.

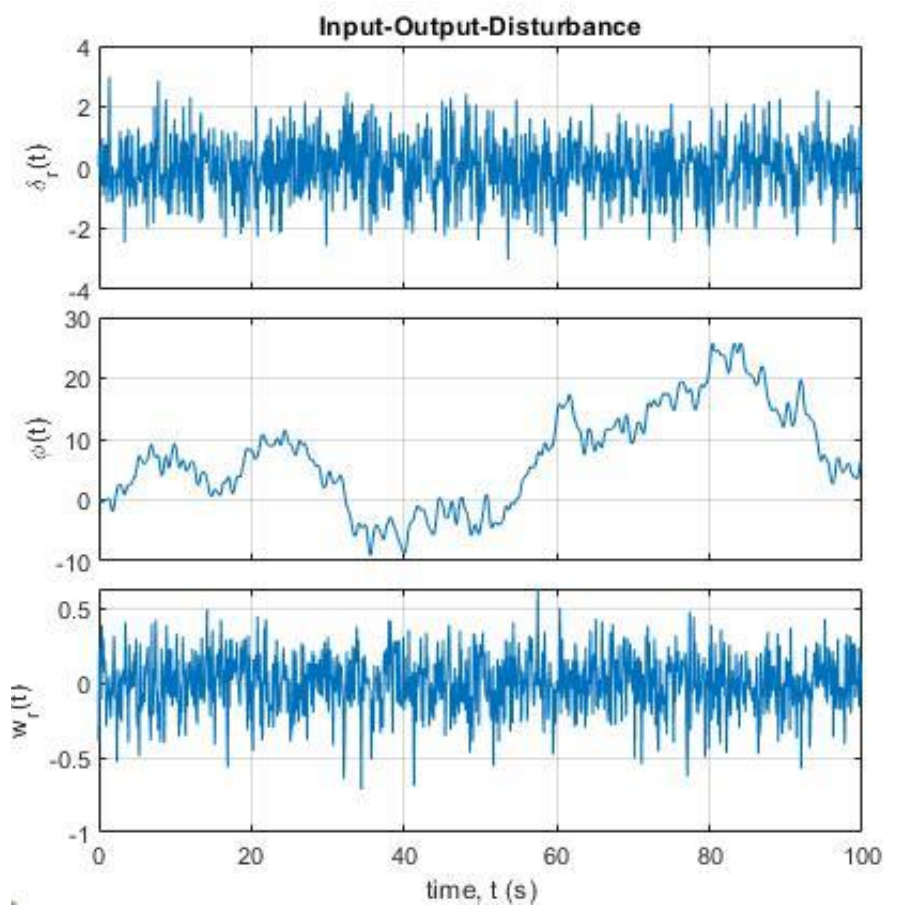

Fig.8. The input-output of fixed-wing UAV with disturbance for rudder input.

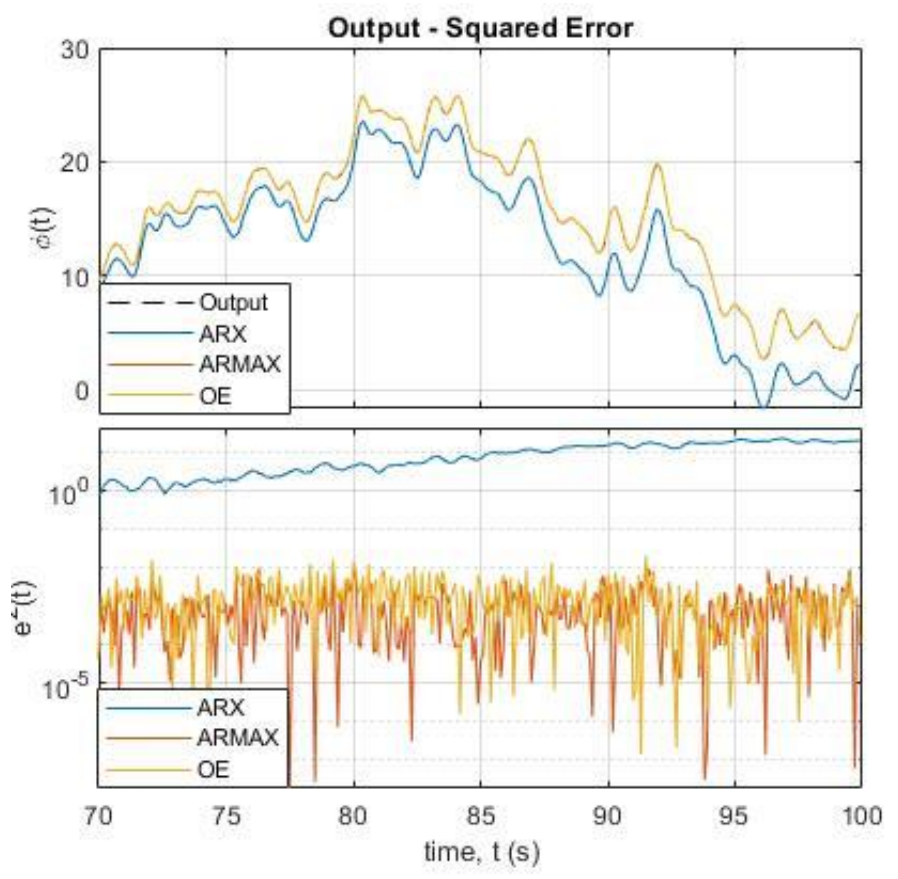

Fig.9. Model outputs and squared errors for rudder input.

The degree of models and estimation ratio system outputrudder input with/without disturbance are presented in Table 2. The maximum parameter estimation accuracy of system without disturbance for rudder input obtained for ( $n a=6, n b=6$ and $n c=6)$ ARMAX and $(n f=6$ and $n b=6)$ OE model. The accuracy of ARMAX and OE model is $99.97 \%$. Also, the maximum accuracy with disturbance system obtained for $(n f=6$ and $n \mathrm{~b}=6$ ) OE model. The accuracy of OE model is $88.36 \%$.

The lateral dynamics of fixed-wing UAV estimated through ARX, ARMAX and OE system identification models. According to results, lateral dynamics of a fixed wing UAV will 
be modelled using parametric system identification approaches. The obtained results also will be basic for the other dynamics of fixed-wing UAVs.

TABLE 2

DEGREE OF MODELS AND ESTIMATION RATIO w/wo DISTURBANCE FOR RUDDER INPUT

\begin{tabular}{|c|c|c|c|}
\multirow{2}{*}{ Model Type } & $\begin{array}{c}\text { Model Degree } \\
\text { ARX }(\boldsymbol{n a}, \boldsymbol{n b}) \\
\text { ARMAX }(\boldsymbol{n} \boldsymbol{a}, \boldsymbol{n b}, \boldsymbol{n c}) \\
\text { OE }(\boldsymbol{n} \boldsymbol{b}, \boldsymbol{n} \boldsymbol{f})\end{array}$ & \multicolumn{2}{|c|}{$\begin{array}{c}\text { Estimation Ratio } \\
\text { w/wo Disturbance } \\
(\%)\end{array}$} \\
\hline ARX & $(4,4)$ & 80.63 & 98.60 \\
\hline ARMAX & $(4,4,4)$ & 86.96 & 99.90 \\
\hline OE & $(4,4)$ & 87.23 & 99.93 \\
\hline ARX & $(5,5)$ & 81.59 & 99.62 \\
\hline ARMAX & $(5,5,5)$ & 87.12 & 99.94 \\
\hline OE & $(5,5)$ & 87.57 & 99.95 \\
\hline ARX & $(6,6)$ & 81.62 & 99.65 \\
\hline ARMAX & $(6,6,6)$ & 88.24 & 99.97 \\
\hline OE & $(6,6)$ & 88.36 & 99.97 \\
\hline
\end{tabular}

\section{CONCLUSION}

The linearized lateral transfer functions of small fixed-wing UAV are used to form input (aileron and rudder) output (roll angle). The dynamic system model is formed as using ARX, ARMAX and OE system identification models and obtained data. The performance of dynamic systems is handled for different noise variances. The results of study show that estimated dynamic models are robust to noise and has minimum model complexity. The maximum estimation accuracy for different noise variances is obtained for OE model. With the help of the model obtained in this study, pre-flight analysis of fixed wing UAV can be performed. Thus, the risks of accidents of fixed wing UAV can be reduced. Thanks to the roll angle obtained with the OE model, the fixed wing UAV will be able to track the specified route with the minimum error. The proposed model will ensure that the payload is carried with high performance to the specified coordinates by tracking a certain route, even under external disturbance, and facilitates safe flight.

\section{REFERENCES}

[1] A. Loya, M. Duraid, K. Maqsood, R. R. Khan. "The implementation and lateral control optimization of a UAV based on phase lead compensator and signal constraint controller." Engineering, vol. 10. 10, 2018.

[2] P. D. Jameson, A. Cooke, "Developing Real-time System Identification for UAVs." 2012 UKACC International Conference on Control, 2012.

[3] W. Saengphet, S. Tantrairatn, C. Thumtae, J. Srisertpol, "Implementation of System Identification and Flight Control System for UAV." $3^{\text {rd }}$ International and Flight Control System for UAV, 2017.

[4] A. Dorobantu, A. Murch, B. Mettler, G. Balas. "System identification for small, low-cost, fixed-wing unmanned aircraft." Journal of Aircraft, vol. 50. 4, 2013, pp.1117-1130.

[5] V. Puttige, S. Anavatti. "Real-time system identification of unmanned aerial vehicles: A multi-network approach." Journal of Computers, vol. 3.7, 2008.

[6] W. Wei, M. B. Tischler, N. Schwartz, K. Cohen. "System identification and flight control of an unmanned quadrotor." Advanced UAV Aerodynamics, Flight Stability and Control: Novel Concepts, Theory and Applications, 2017, pp.695-727.
[7] A. Altan, R. Hacioğlu. "Model predictive control of three-axis gimbal system mounted on UAV for real-time target tracking under external disturbances." Mechanical Systems and Signal Processing, 2020.

[8] A. Altan, R. Hacioğlu, "Modeling of Three-axis Gimbal System on Unmanned Air Vehicle (UAV) under External Disturbances." 2017 25th Signal Processing and Communications Applications Conference (SIU), 2017.

[9] A. Altan, Ö. Aslan, R. Hacioğlu, "Model Predictive Control of Load Transporting System on Unmanned Aerial Vehicle (UAV)." Fifth International Conference on Advances in Mechanical and Robotics Engineering, 2017.

[10] Y. H. Aleed, T. A. Tutunji, "RC Helicopter Modeling Using Reengineering and System Identification." 14th International MultiConference on Systems, Signals \& Devices (SSD), 2017.

[11] C. Dube, J. O. Pedro. "Modelling and closed-loop system identification of a quadrotor-based aerial manipulator." Journal of Physics: Conference Series, vol. 1016.1, 2018.

[12] J. E. Sierra, M. Santos. "Modelling engineering systems using analytical and neural techniques: Hybridization." Neurocomputing, 2018, pp. 7083.

[13] A. E. Ahmed, A. Hafez, A. N. Ouda, H. E. H. Ahmed, H. M. AbdElkader. "Modeling of a small unmanned aerial vehicle." International Journal of Aerospace and Mechanical Engineering, vol. 9. 3, 2015.

[14] L. Ljung, System Identification, System Identification Theory for The Users, 1999, p. 609.

\section{BIOGRAPHIES}

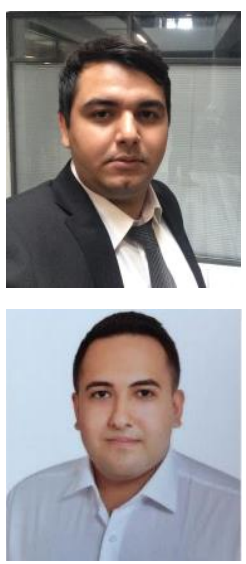

EGEMEN BELGE received his B.Sc. and M.Sc. degrees both in Electrical Electronics Engineering from the University of Mersin in 2016 and 2018, respectively. $\mathrm{He}$ is currently Ph.D. student at Zonguldak Bülent Ecevit University, Zonguldak, since 2018 where he works as a research assistant.

HIZIR KAAN KABA received his B.Sc. degree in Mechatronics Engineering from the University of Iş1k in 2018. He is currently M.Sc. student at Zonguldak Bülent Ecevit University.

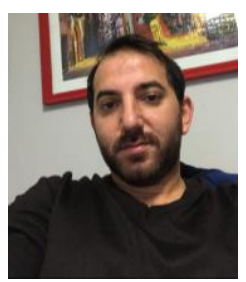

AHMET PARLAK received his B.Sc. degree in Electrical Electronics Engineering from the University of Zonguldak Bülent Ecevit University in 2016. He is currently M.Sc. student at Zonguldak Bülent Ecevit University.

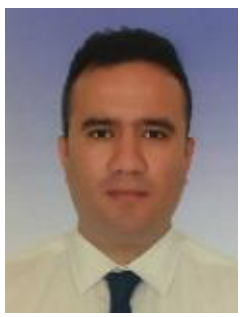

AYTAÇ ALTAN recieved his B.Sc. and M.Sc. degrees in the department of Electrical Electronics Engineering from Anadolu University in 2004 and 2006, respectively. $\mathrm{He}$ is received his Ph.D. degree in the department of Electrical Electronics Engineering from Zonguldak Bülent Ecevit University in 2018. He is currently a lecturer at the department of Electrical Electronics Engineering at the Zonguldak Bülent Ecevit University in Turkey. His research interests include system identification, signal processing, image processing, model based control and robotic systems. 


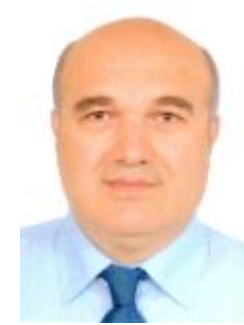

RIFAT HACIOĞLU received his B.Sc. degree in the department of Electrical Electronics Engineering from Dokuz Eylül University in 1993. He is received his M.Sc. and Ph.D., degrees in the department of Electrical and Computer Engineering from Illinois Institute of Technology, Chicago, USA, in 1996 and 2002, respectively. He is currently an Associate Professor at the department of Electrical Electronics Engineering at the Zonguldak Bülent Ecevit University in Turkey. His research interests are signal processing, control applications, linear and nonlinear system identification, parametric model estimation approaches, microprocessor based control systems, image identification problems. 